\title{
Challenges Associated with Implementing 5G in Manufacturing
}

\author{
Eoin $\mathrm{O}^{\prime}$ Connell ${ }^{1,2, *(\mathbb{D})}$, Denis Moore ${ }^{1}$ and Thomas Newe ${ }^{1,2, *(\mathbb{D})}$ \\ 1 Electronic \& Computer Engineering Department, University of Limerick, V94 T9PX Limerick, Ireland; \\ denis.moore@ul.ie \\ 2 CONFIRM Centre for Smart Manufacturing, University of Limerick, V94 T9PX Limerick, Ireland \\ * Correspondence: eoin.oconnell@ul.ie (E.O.); thomas.newe@ul.ie (T.N.)
}

Received: 24 April 2020; Accepted: 9 June 2020; Published: 12 June 2020

\begin{abstract}
G networks will change several industries, including manufacturing. 5G has the potential to become the future communication platform of choice for many industries and in particular the manufacturing sector, driving the future of Industry 4.0 and smart manufacturing. The vision of a "factory of the future" is now tangible for many industry sectors. The ability to cope with increased bandwidth, latency requirements, big-data generated from more connected equipment and the data processing required on the factory floor is a massive challenge for industry. This paper discusses how 5G can impact a manufacturing environment, the standards and technical requirements needed to meet the demands of utilizing 5G, and the security issues that need to be addressed if planning a $5 \mathrm{G}$ deployment.
\end{abstract}

Keywords: Internet of Things; 5G; spectrum; manufacturing; private network; edge; core; MPN; $5 \mathrm{G}$ security; industry 4.0; smart manufacturing

\section{Introduction}

$5 \mathrm{G}$ promises to deliver faster download speeds, lower latency and higher capacity, as shown in Table 1; Table 2 below. The goal of Industry 4.0 is to maximize efficiency by utilizing these features across all processes and assets at all times; to provide a better understanding of the manufacturing processes across their manufacturing sites in near real-time. With the expected increase in data requirements ranging from mission-critical to massive machine connectivity, the deployment of $5 \mathrm{G}$ has raised expectations that it will open new opportunities for manufacturing business models.

Table 1. Data Speeds for 3G, $4 \mathrm{G}$ and 5G.

\begin{tabular}{cccc}
\hline Network Type & Average Download Speeds & Peak Download Speeds & Theoretical Download Speeds \\
\hline $3 G$ & $8 \mathrm{Mbps}$ & $\sim 20 \mathrm{Mbps}$ & $42 \mathrm{Mbps}$ \\
\hline $4 \mathrm{G}$ & $32.5 \mathrm{Mbps}$ & $90+\mathrm{Mbps}$ & $300 \mathrm{Mbps}$ \\
\hline $5 \mathrm{G}$ & $130 \mathrm{Mbps}-240 \mathrm{Mbps}$ & $599 \mathrm{Mbps}+$ & $10-50 \mathrm{Gbps}$ \\
\hline
\end{tabular}

Table 2. Comparison of 3G/4G and 5G latency times [1].

\begin{tabular}{cc}
\hline Network Type & Milliseconds (ms) \\
\hline 3G Network & 60 ms (Typical) \\
\hline 4G Network & 50 ms (Typical) \\
\hline 5G Network & 1 ms (theoretical) \\
\hline
\end{tabular}


According to McCann et al. [2], “The Fourth Industrial Revolution or simply 'Industry 4.0' is how manufacturing industry expects to maximise the innovations of $5 \mathrm{G}$ wireless communications by automating industrial technologies and utilising other enabling technologies such as artificial intelligence (AI) and machine learning. Industry expects this to lead to more accurate decision making such as automation of physical tasks based on historical information and knowledge, or improved outcomes for a wide range of vertical marketplaces not just in manufacturing but verticals such as agriculture, supply chain logistics, healthcare, energy management and an ever-increasing number of industries becoming more aware of the potentials of 5G." It is expected to achieve this in three ways through the use of network slicing, Figure 1.

- $\quad$ eMBB (Enhanced Mobile Broadband)

- $\quad$ URLLC (Ultra-Reliable Low Latency Communications)

- mMTC (Massive Machine-Type Communications)

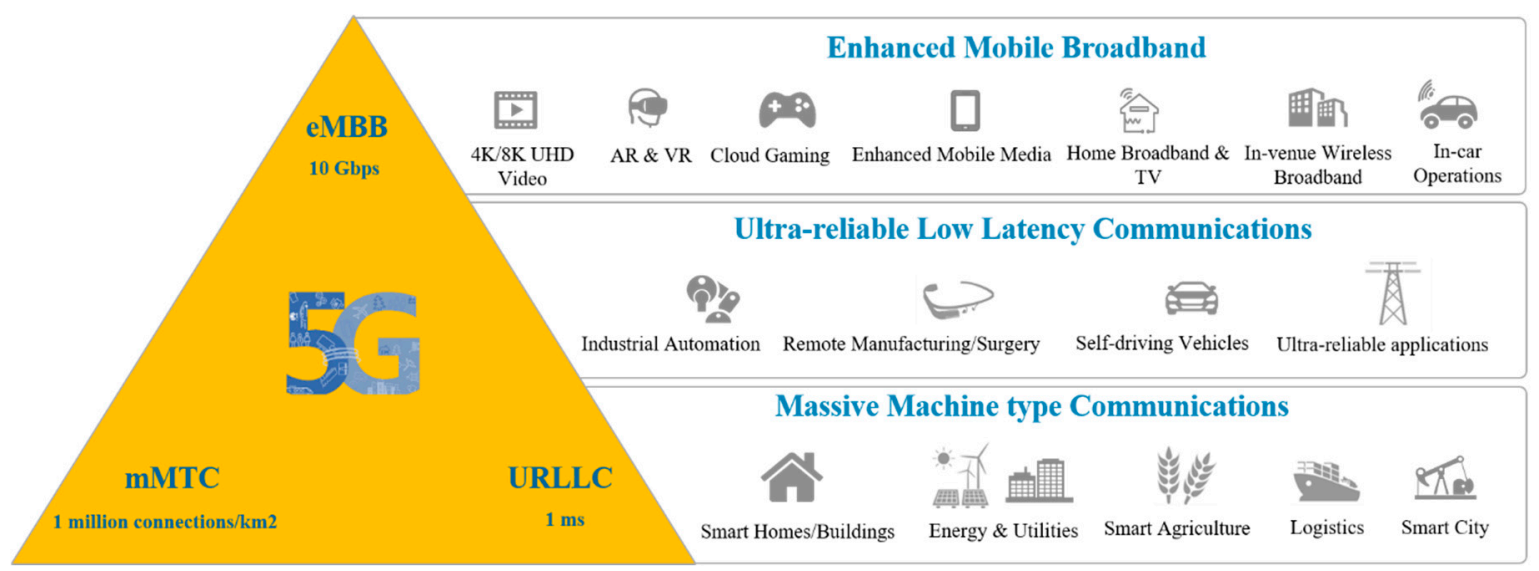

Figure 1. 5G key usage scenarios.

\subsection{Enhanced Mobile Broadband (eMBB)}

eMBB can initially be addressed by the expansion to existing $4 \mathrm{G}$ services as it aims to service more densely populated metropolitan centers with downlink speeds approaching $1 \mathrm{Gbps}$ (gigabits-per-second) indoors, and $300 \mathrm{Mbps}$ (megabits-per-second) outdoors. One way to accomplish this is through the installation of extremely high-frequency millimeter-wave (mm-Wave) antennas. For areas that are more urban outlying and beyond into rural setting areas, eMBB will work towards replacing 4G's current LTE (Long-Term Evolution) scheme, with a new network of lower-power omnidirectional antennas providing $50 \mathrm{Mbps}$ downlink service. eMBB traffic can be deemed to be an addition to the $4 \mathrm{G}$ broadband service currently available. It is capable of large payloads and a by-a-device activation pattern that is capable of remaining stable over an extended time interval. This allows the network to schedule wireless resources to the eMBB enabled devices such that no two eMBB devices compete or access the same resource simultaneously [3]. To provide these, it is expected that $5 \mathrm{G}$ will deliver:

- Traffic capacity of up to $10 \mathrm{Mbps}$ per sq. metre in hotspot areas.

- Data transfer rates the user experiences of up to $1 \mathrm{Gbps}$ with peak data rates of transfer in the tens of Gbps and a capacity of maximum traffic volume of at least 1 Tbps per sq. kilometre.

- Latency as low as $1 \mathrm{~ms}$ for user experience levels of data exchange.

- Connection density of up to one million connections per sq. kilometre.

- High mobility, facilitating connectivity up to $500 \mathrm{~km} / \mathrm{h}$ in high-speed trains and up to $1000 \mathrm{~km} / \mathrm{h}$ in aeroplanes, with enhanced user experience. 


\subsection{Ultra-Reliable and Low-Latency Communications (URLLC)}

URLLC can address critical needs of communications where bandwidth is not quite as crucial as speed, e.g., an end-to-end latency of $1 \mathrm{~ms}$ or less. The design of a low-latency and high-reliability service involves several components, including, incredibly fast data turnaround, efficient control and data resource sharing, grant-free based uplink transmission, and advanced channel coding schemes. Uplink grant-free structures guarantee a reduction in user equipment (UE) latency transmission by avoiding the middle-man process of acquiring a dedicated scheduling grant. These services are supported by the 5G New Radio (NR) standard. URLLC should be the tier that addresses the autonomous vehicle category, where decision time for a reaction to a possible accident needs to be almost non-existent. URLLC makes 5G a possible competing solution with satellite, for Global Positioning System (GPS) geolocation services [4,5].

\subsection{Massive Machine-Type Communications (mMTC)}

5 G offers massive machine-type communication (mMTC), which aims to support tens of billions of network-enabled devices to be wirelessly connected [1]. Today's communication systems already serve many MTC applications. However, the characteristic properties of mMTC, i.e., the massive number of devices and the tiny payload sizes, require novel approaches and concepts. $5 \mathrm{G}$ allows a density of one million devices per square kilometer $[3,6]$. 5G will be able to carry a lot more data and transfer it much faster than $4 \mathrm{G}$ LTE, however, faster is not always better or even necessary in the Internet of Things (IoT) world, especially when it typically requires more power on the end device. So the 5G NR standard will introduce new device types, like Cat-M1 (operates at 1.4 MHz bandwidth) and narrow band (NB-IoT). Both NB-IoT and Cat-M1 devices can sleep for extended periods of time with extended discontinuous reception (eDRX) and power-saving mode (PSM) functionalities, which greatly reduce device power consumption This will enable lower-power, and battery-driven devices to be utilized. 5G in industrial automation will facilitate real-time wireless sensor networks with location and asset tracking. 5G networks will eventually be able to supersede wired connections in even the most demanding of applications, such as automation control and high throughput vision systems $[7,8]$.

This paper differs from previous publications in this space in that it provides an overview of $5 \mathrm{G}$ and the technology required, how it will positively impact the future of the manufacturing sector, enabling it to move to industry 4.0 and the infrastructure requirement to enable an industry to adopt 5G. The paper is structured as follows: Section 2 discusses the potential and impact of 5G in manufacturing; Section 3 presents the use cases of $5 \mathrm{G}$ in manufacturing while Section 4 lists the infrastructure requirements for a 5G network; Section 5 discusses where 5G lies in the wireless spectrum and Section 6 outlines the concept and benefits of private $5 \mathrm{G}$ networks; Section 7 briefly discuss $5 \mathrm{G}$ security issues and Section 8 the 5G standard; Finally our conclusions regarding $5 \mathrm{G}$ and its role in manufacturing are presented.

\section{The Potential of 5G in Manufacturing}

$5 \mathrm{G}$ has the potential to become the underlying digital fabric connecting all elements of the manufacturing world. 5G promises fast connectivity, more bandwidth than Wi-Fi and 4G LTE but also low latency with support for thousands of devices in a small location, all of which are attractive prospects to manufacturing facilities. 5G's integration capability with time-sensitive networking (TSN) makes smart factories an achievable goal. TSN capability will help 5G avail of the enhancements to the ethernet to make it more reactive to demand or "deterministic", essentially making 5G available on time with low latency, low jitter and high reliability. In order for this interface to achieve low latency, all the devices have to synchronize to the same time-base, in effect they have to be 'time aware'.

Widespread rollout of $5 \mathrm{G}$ is now a reality. Device manufacturers have already started deploying $5 \mathrm{G}$ network equipment while network carrier companies are on the verge of commercializing $5 \mathrm{G}$ networks in new verticals such as manufacturing [9]. While there are several barriers still impeding the universal deployment of $5 \mathrm{G}$ networks, there are also endless applications and potential benefits. 
$5 \mathrm{G}$ has the potential to revolutionize various critical areas such as the IoT, entertainment, automation, IT, and more. 5G private networks, which offers several advantages, will boost the power of Industrial IoT (IIoT) as 5G offers more than increased network speeds and bandwidth [10].

$5 \mathrm{G}$ wireless technology and its associated deployment is being implemented at a time when many industries are themselves transforming through greater automation, using techniques such as the IoT, machine vision, machine learning and robotics. 5G connections will go beyond human communication needs and is expected to enable the intelligent internet of things. The next generation (NextGen) of telecommunication technologies will be adopted by a broader range of industries and sectors with digital manufacturing a big beneficiary. NextGen wireless control of highly sophisticated mobile machines is becoming feasible, and it will allow the possibility for machines to take advantage of the enormous computing power and cloud storage options available, without being tethered by physical wires. An early area of interest for $5 \mathrm{G}$ in the IIOT is to facilitate factory monitoring, where sensors are installed in the factory to monitor equipment conditions and to oversee that everything is working as it should, and to identify potential issues before they occur. These sensors will be interrogated and the end data used to create insights into end products and deliverables, providing improvements in quality control and bespoke manufacturing. 5G has incorporated many requests to improve 4G and now is capable of delivering new services that may be tuned to the requirements of a customers' business models (Figure 2) e.g., manufacturing [7,8].

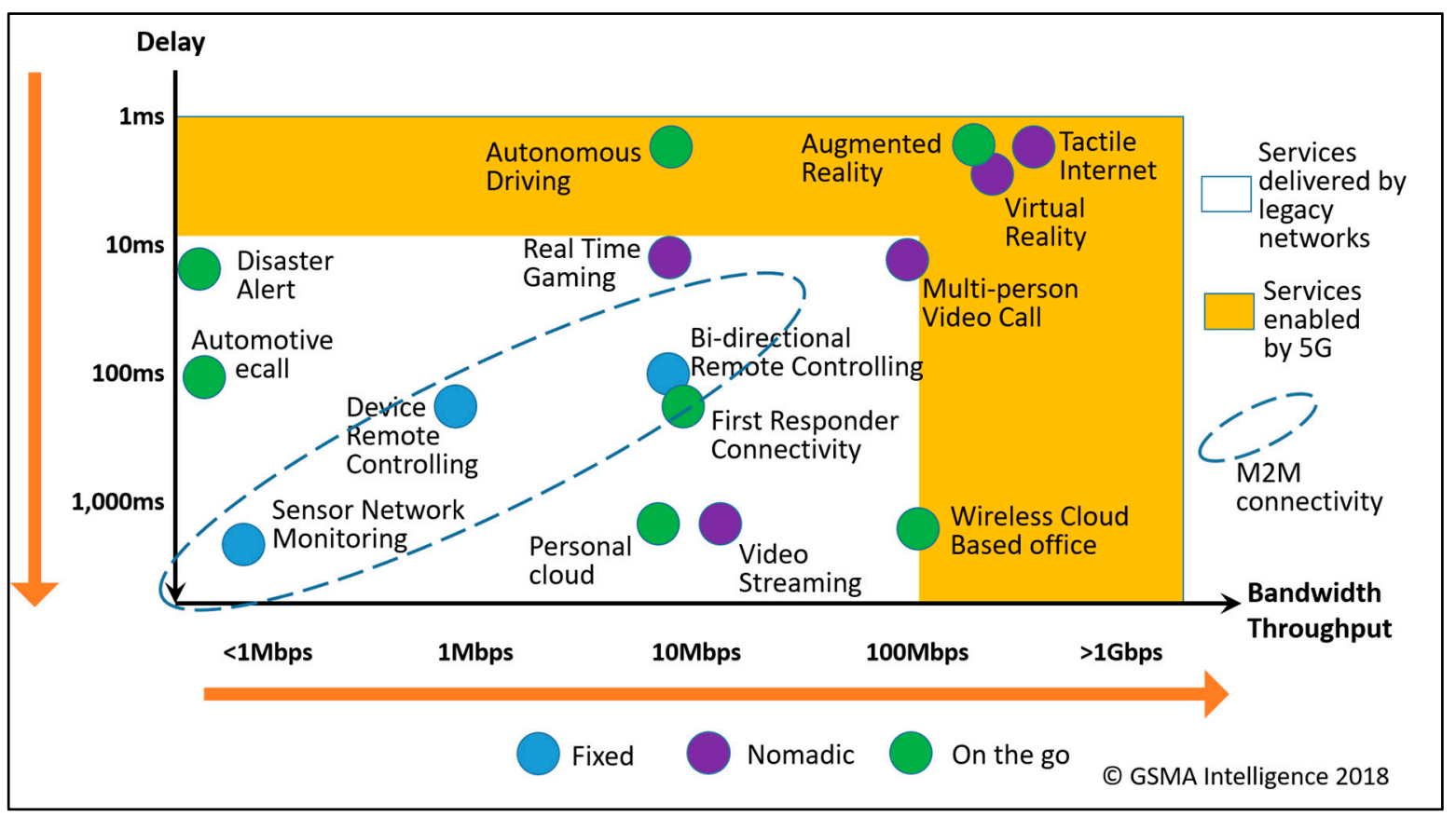

Figure 2. Use cases-latency and bandwidth requirements [8].

Data rates of $10 \mathrm{Gbps}$ are widely accepted as a feasible expectation for 5G when it is fully commercially available, but early 5G services seem to be maxing out at approximately 1 Gbps [11]. As deployments increase, all the data generated will be collected and analyzed to give detailed insights into every facet of a factory's operation. Machine learning-capable smart robots will build products and be enabled to move goods and deploy equipment in and around the factory, generating even more data. Manufacturing is expected to pivot from an assembly line to the production of highly customized products essentially enabling the mass production of bespoke products, with workers streaming virtual/augmented reality videos or working in virtual environments [12]. These concepts can be facilitated by the 5G NR standard.

While the range of benefits of $5 \mathrm{G}$ is impressive, there are limitations inherent in the deployment of $5 \mathrm{G}$. The spectrum allocated is at a higher frequency, and proportionately the wavelength of those 
signals is smaller, which is limiting to the signal penetration capability. 5G will initially be allocated spectrum at the 3 to $6 \mathrm{GHz}$ range (depending on national regulations). Within a heavy engineering environment, with dense, generally metallic machinery, signal penetration should be factored into the design. To calculate the penetration of a 5G signal the wavelength of the signal is needed, as signal penetration is proportional to its wavelength, i.e., longer wavelengths penetrate obstacles better. This is calculated by using the frequency and the speed of light (in the medium), in Equation (1) [13].

$$
\lambda=\mathrm{c} / \mathrm{f}
$$

where;

$\lambda($ Lambda $)=$ Wavelength in meters

c $=$ Speed of Light $(299,792,458 \mathrm{~m} / \mathrm{s})$

$\mathbf{f}=$ Frequency $(\mathrm{MHz})$

Applying the formula above, it becomes clear that there may be issues around signal penetration and distance issues when transmitting at the bands allocated for 5G. As signal penetration is directly related to signal wavelength, shorter wavelengths are more easily reflected or refracted. As the frequencies utilized by $5 \mathrm{G}$ increases, the signal penetration decreases, i.e., it is more susceptible to attenuation. Accordingly, manufacturing should choose their operating band wisely as it may have a significant impact. A summary comparison of 5G and 4G LTE (Long-Term Evolution) is shown below in Figure 3.

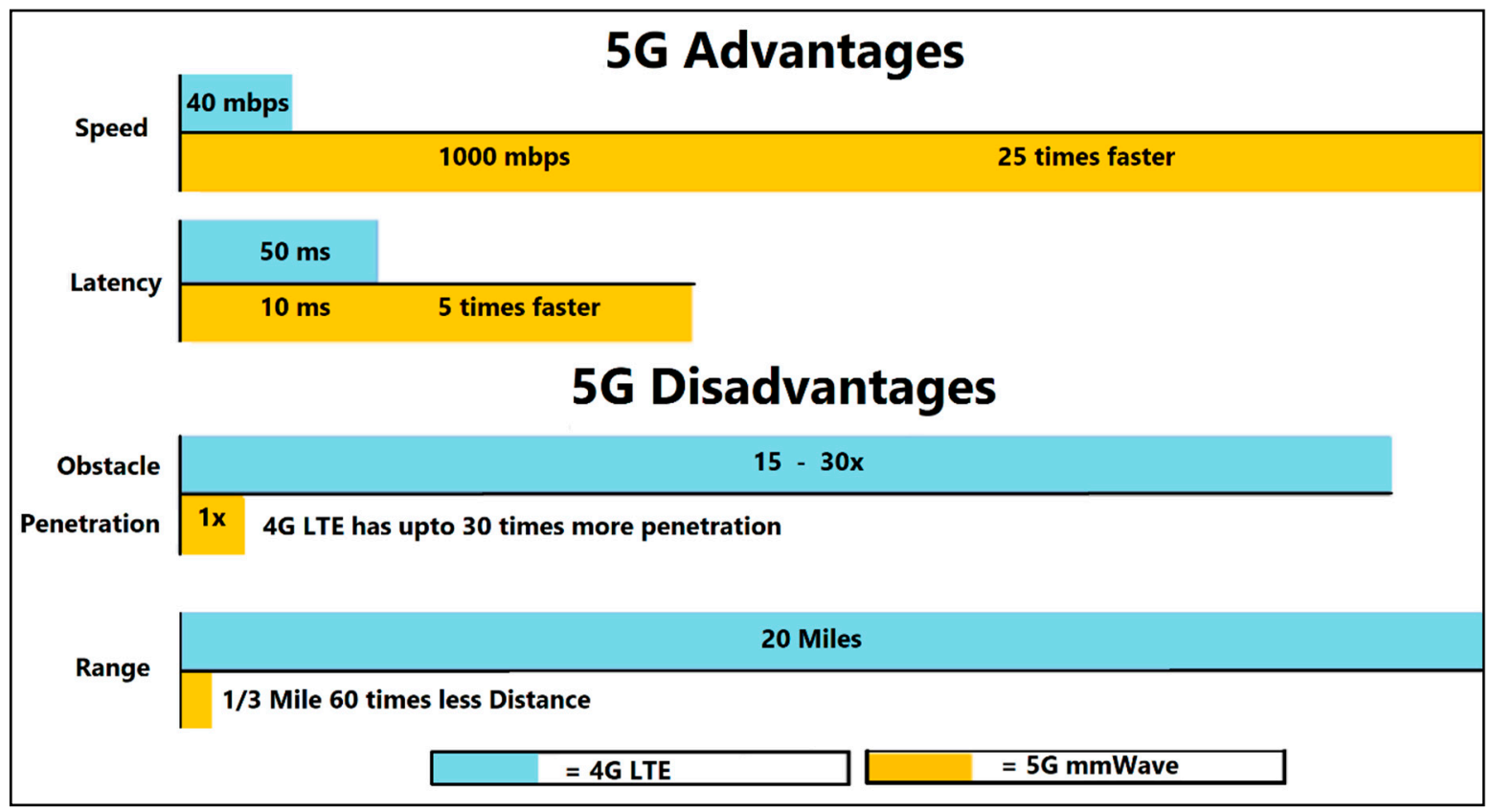

Figure 3. Comparison of $4 \mathrm{G}$ LTE versus $5 \mathrm{G}$.

\section{How 5G Will Impact Manufacturing}

For 5G to be deployed successfully in a manufacturing environment, there must be close collaboration between all systems, spanning across the corporate information and communication technologies (IT) and the manufacturing operational technologies (OT) to maximize the capabilities inherent in 5G. The vision is that industry will be able to construct cyber-physical systems that enable precision control in near real time, from virtually anywhere in the world [14]. Typical deployment scenarios, some of which were mentioned previously, are given below: 
- Ability to remotely operate equipment e.g., production line robotics on the factory floor. Typical applications being welding, painting and assembly.

- Remote control of supply chain equipment e.g., operator can remote control equipment such as untethered robots, typical applications include, autonomous ground vehicles (AGVs) or forklifts.

- Remote monitoring of equipment e.g., the transmission of diagnostics information, so service technicians arrive prepared for successful repairs and updates when needed.

- Machine to machine communication: closed-loop communications between machines to optimize manufacturing processes.

- Intra- and inter-enterprise communication: for monitoring of assets distributed in broader geographical areas across the value chain.

- Augmented reality support in design, maintenance and repair: use of augmented reality to aid in the execution of procedural tasks in the design, maintenance and repair domain (through simulations).

Each industry deployment has to look at multiple constraints and identify a solution that will deliver an optimal solution to meet the business requirements of the end-user or corporate need.

The main challenges for industry adoption of $5 \mathrm{G}$ are:

- Cost: the manufacturing industry has high-cost reduction requirements and will only implement new applications if these have been proven to reduce costs ultimately.

- Safety: hundreds of connected automated devices on a factory floor can create a hazardous environment for humans.

- Deployment knowledge: many small and medium enterprises (SMEs) do not have the capacity to resource the learning requirements or the technical ability to capitalise on the potentials of 5G.

- RF interference: several objects on the factory floor are already using radio communications.

Another consideration for manufacturing is the increased proliferation of more sophisticated collaborative robots (cobots). These smaller, lightweight, smart robots can detect when a human is nearby, making it possible for that individual to work more safely alongside the machine. A typical usage scenario for a cobot might be to take care of such tasks as moving heavy parts or doing repetitive tasks, while a human collaborator handles the more advanced tasks. Cobots are already on the market, but $5 \mathrm{G}$ networks should help make them more useful. An example of adding value is the capitalizing on the lower latency of $5 \mathrm{G}$ which will allow a more instantaneous real-time presence awareness that would enable the robot to work at faster speeds next to a human with a reduced risk (close to zero) of injury $[15,16]$.

\section{Use Cases of 5G in Manufacturing}

While $3 \mathrm{G}$ and $4 \mathrm{G}$ offer stepped improvements in speed and bandwidth, $5 \mathrm{G}$ will be the first cellular platform to provide reliable machine-to-machine and Industrial IoT systems. There are many potential use cases for 5G in Manufacturing.

\subsection{Supply Chain Unification}

Digitalizing the modern manufacturing unit, introducing a higher degree of automation control and the advent of smart Internet of Things devices is a focal point of the fourth industrial revolution. The introduction of $5 \mathrm{G}$ into the manufacturing industry enables supply chains to pivot from a dispersed series of independently managed location networks to an increasingly connected ecosystem of devices that share knowledge across the network in pseudo real-time. As manufacturing units become more capable with the integration of 5G into smart devices, the manufacturing industry can leverage the advanced communication capabilities between devices, unifying the production process. 5G is rapidly becoming the connection of choice, serving as the intermediary medium of data transfer between every component of the manufacturing ecosystem [17]. 


\subsection{Artificial Intelligence (AI) and Fast Decision Making}

The best-run processing plants depend on immense information pools to decide-with inescapable deferrals as information is gathered, cleaned, and investigated. 5G accelerates the choice process duration, permitting enormous measures of data to be ingested, handled and actioned close to real-time. In a few substantial enterprises, for instance, producers have had the option to sell overabundance vitality back to the matrix when machines are not running, and costs are reasonable. $5 \mathrm{G}$ will permit the gushing of information progressively to the cloud, and the utilization of live video examination. For instance, a surveillance camera could see an unsettling influence, recognize if there is a fast-approaching risk or threat via $\mathrm{AI}$, and dispatch an automaton or alarm a laborer to examine.

Modern-day manufacturing is based on multiple technologies working together, which translates to the creation of large data sets being created. This large increase in data collection can be transmitted and analyzed faster with 5G's low latency coupled with its high bandwidth capacity. The expansion of multiple sensors to machine monitoring by industrial facilities is creating more information than in the past. Transmission through wired systems is costly compared to wireless networks. 5G can help by empowering the utilization of up-to-date information at scale [18]. While the production floor is the obvious point at which manufacturing can avail of the benefits of 5G there are also other examples of how $5 \mathrm{G}$ can contribute to the manufacturing environment in a non-direct manner. Examples of areas within an organization that could benefit indirectly are listed in Table 3.

Table 3. Use cases in manufacturing [2].

\begin{tabular}{|c|c|c|}
\hline Use-Case Category & Scenario & Impact \\
\hline Time-critical processes & $\begin{array}{ll}\text { - } & \text { Real-time, closed-loop } \\
\text { robotic control } \\
\text { - } \\
\text { Video-driven } \\
\text { machine-human interaction } \\
\text { Augmented Reality/ Virtual } \\
\text { Reality (AR/VR) for } \\
\text { maintenance and training }\end{array}$ & Increased efficiency and yields; safety \\
\hline Non real-time processes inside the factory & $\begin{array}{l}\text { - Tracking products and } \\
\text { machine inventory } \\
\text { - } \\
\text { - } \\
\text { Ron-real-time sensor data } \\
\text { and diagnostics }\end{array}$ & $\begin{array}{l}\text { Optimized management of } \\
\text { production facilities }\end{array}$ \\
\hline Enterprise communication & $\begin{array}{l}\text { - } \quad \text { Logistics and warehousing } \\
\text { Employee and } \\
\text { back-office communications } \\
\text { - Tracking } \\
\text { goods post-production }\end{array}$ & Improved business operations \\
\hline
\end{tabular}

\subsection{Cloud Control of Machines}

The capabilities afforded by $5 \mathrm{G}$ will enable manufacturers to design robots that benefit from increased data integration and real-time decision making. For a considerable length of time, manufacturing plant computerization has depended on programmable logic controllers (PLCs) that were introduced to control the machines they were connected to and afterwards hardwired into computing systems to implement control management. Cloud control management is mostly virtualized computing resources, storage, applications, and services that are managed by software so the data generated can be accessed on-demand. Cloud management is a combination of software, automation, policies, governance, and algorithms that determine how those cloud computing services are offered. 
When 5G reliably meets its initial specifications, the PLC will be able to be virtualized in the cloud, empowering machines to be controlled remotely and continuously with reduced expense to the company or end-user.

\subsection{Expanded Reality}

Factories are home to multiple advanced technological solutions designed to overcome many problems; one such solution is virtual reality/augmented reality. Manufacturers who have been skeptical of implementing augmented and virtual reality technologies will be able to take full advantage and leverage them for real-time simulations and predictive maintenance. $5 \mathrm{G}$ connectivity has the potential to unleash massive amounts of innovation in virtual experiences that require low latency and ultra-fast data speeds. The stability and low latency that 5G networks will bring and the use of a range of frequencies, as well as advancements within both hardware and software, is making industry very excited about the future use cases that will utilize this technology. One of the most pertinent issues facing manufacturers is the loss of skilled workers as manufacturing transitions towards digitalization. Virtual reality/augmented reality provide a way to minimize the loss of the expertise of experienced workers by placing them in the mentoring role of remote experts capable of guiding newer technicians and engineers.

5G enables manufacturing organizations to extract the potential of augmented reality solutions, ultimately leading to increased productivity. Equipment maintenance is crucial to the smooth running of most factories. The unexpected breakdown of equipment can cost companies significant amounts depending on how long the process is halted. Engineers can use the historical data to counteract this, but this alone will still lead to excess downtime, e.g., the use of smart glasses will allow access to the constantly generated equipment data which will allow more in-depth analysis to highlight problem equipment well before it fails. This will allow companies to choose when to maintain the equipment to minimize the financial impact. Repair manuals can be accessed in real-time, and a walkthrough of how to get access to or remove and maintain parts can be displayed to the technician or engineer. This will lead to more efficient repairs and significantly less mentoring for training staff. The best information and work guidelines can, in this manner, be imparted to all relevant staff precisely when they need it, building specialist abilities all the more rapidly, securely, and successfully than at any other time.

\section{Infrastructure Requirements for $5 \mathrm{G}$}

According to Singh et al. [19], attempts have been made to characterize the broad set of requirements of industrial automation. The integration of $5 \mathrm{G}$ to an existing network architecture will require the addition of an onsite $5 \mathrm{G}$ network comprised of macro or small cells, which will need to be fully IP-enabled. If an existing private $4 \mathrm{G}$ network is in situ, the $4 \mathrm{G}$ backhaul network can be reused whenever available and possible, but backhaul capacity will likely need to be upgraded since the 5G network delivers far more traffic than any cellular network did before. 5G mobile networks will significantly affect both the wireless side and the wired side of network infrastructure requirements due to the increased data traversing to and from the wired and wireless networks. The smart factory is represented below in Figure 4 by being divided into several layers with different network choices at each layer; each network has different demands and rates the importance of various properties differently. It is decisions at each of these levels that will dictate the level of integration that $5 \mathrm{G}$ can bring to manufacturing. The key new concept of network slicing in 5G will enable tenants to gain different levels of connectivity from their service provider to accommodate various use cases. To achieve this network slicing, 5G will be an all-cloud architecture and this will ultimately require the use of software defined networking (SDN) and network function virtualization (NFV) [20].

Future 5G networks will have to provide a significantly higher system capacity than today and solve the anticipated spectrum crunch. There will be many infrastructure improvements required to provide a $5 \mathrm{G}$ network within a manufacturing environment. Requirements will range from the 
upgrading of backbone infrastructure within the organization to managing the utilization of the following:

- Spectrum adoption;

- Fiber rollout internally (10 Gb minimally);

- High-speed switches and routers;

- On-site computing;

- High-speed uplink to cloud computing facilities;

- Deploying edge-connecting devices.

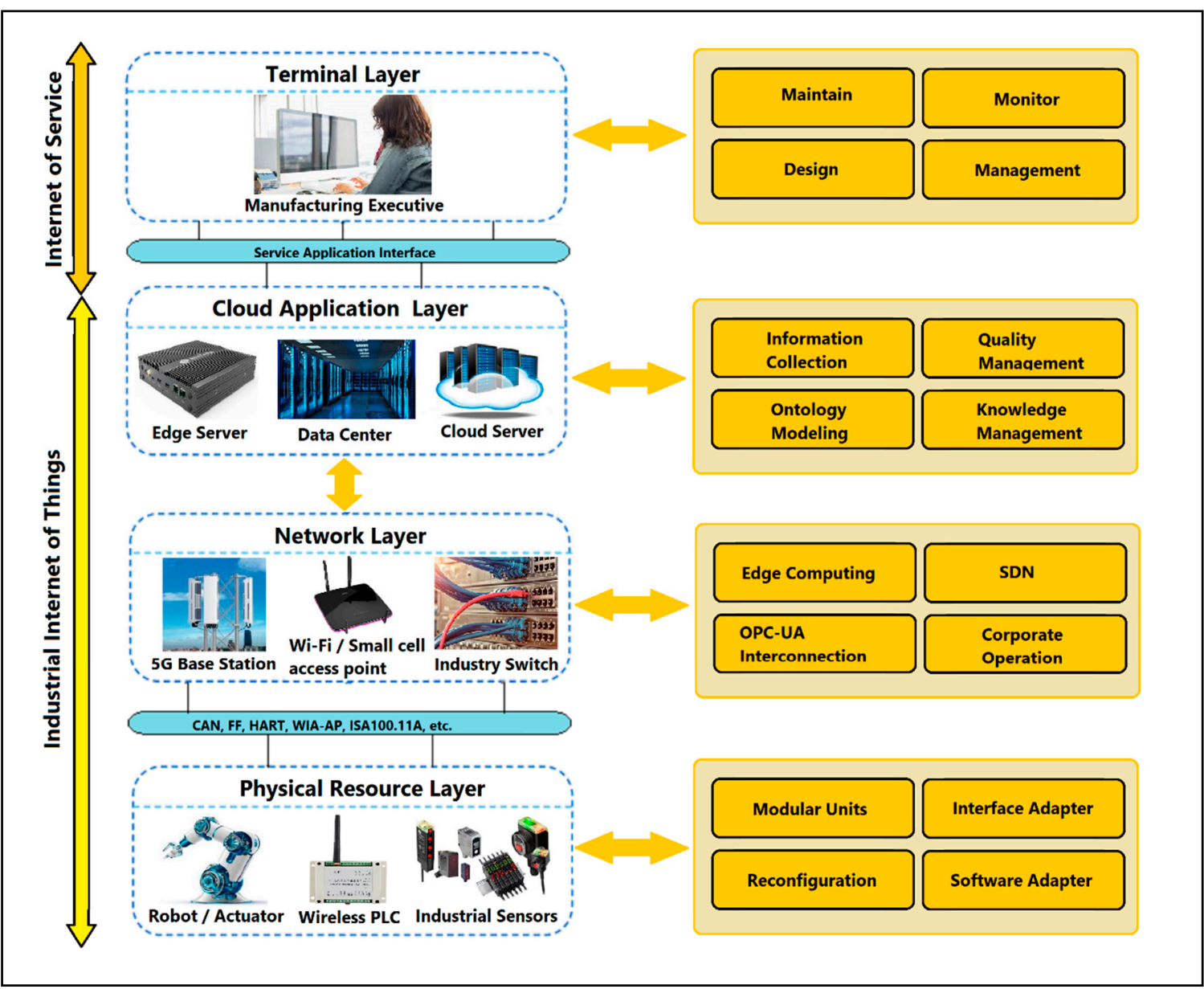

Figure 4. Hierarchical architecture for smart factory [21].

A specific challenge will be the analysis of the placement of network intelligence either at the edge, in the fog, or in a private/public cloud. The precise locating of edge analytics anywhere from the network edge up to the edge cloud computing (ECC) will be a challenge for many sectors. To progress the development of the digital factory, there is a need for a robust wireless network that can transfer large amounts of data fast. Reliable low latency and high data-transfer rates are crucial in connecting smart devices in Industry 4.0. The prospect of download speeds between $1 \mathrm{Gbps}$ and $10 \mathrm{Gbps}$, and upload speed or latency of just 1 millisecond, makes the prospect of a $5 \mathrm{G}$ network fascinating for industry, but the reality is that typically $5 \mathrm{G}$ users will not achieve anything near the theoretical top speeds. The actual rate of a 5G connection will depend on many factors including location, network connection type (private or via a TELephone Company, TELCO), how many other devices are connecting, and the specifications of the device being used. The aim for manufacturing has to be 
achieving a minimum download speed of $50 \mathrm{Mbps}$ and latency of $10 \mathrm{~ms}$ in a scalable manner which will represent a significant improvement over current rates.

\subsection{G in a Heterogeneous Network Infrastructure}

Future $5 \mathrm{G}$ networks will have to provide a significantly higher system capacity than today and solve the anticipated spectrum crunch. Communications regulators globally are managing the allocation of spectrum for $5 \mathrm{G}$ usage; typically, the spectrum is being auctioned to telephone companies (Telcos) who are then offering a range of solutions to industry. In Europe, three major frequency bands have been made available for $5 \mathrm{G}$ use. These three bands are $700 \mathrm{MHz}, 3.6 \mathrm{GHz}$ and $26 \mathrm{GHz}$. It should be noted that spectrum regulators in some countries have started the process of opening up shared spectrum for local use in specific bands, which will enable the deployment of private $5 \mathrm{G}$ networks and enable easier research and development deployments. For example, the regulator in the USA opened up the $3.5 \mathrm{GHz}$ band as of January 2020 for commercial use known as the CBRS (Citizens Broadband Radio Service) band.

In addition to cellular advances, Wi-Fi networking is also getting significant upgrades and this supplementing technology will add to the $5 \mathrm{G}$ ecosystem. Each technology offers individual improvements; they will also work in tandem to fill in gaps in environments where other technologies may not be ideal. The Wi-Fi Alliance is referring to the IEEE 802.11ax specification as Wi-Fi 6 because it's the sixth generation of Wi-Fi.

Harnessing 5G's capabilities in terms of security, synchronizing and network slicing times between devices will be a challenge for industry. These challenges are expected to take some time to be solved. Therefore, while $5 \mathrm{G}$ should be seen as the solution going forward, the timeline to address those issues should make companies plan a transition approach to adopting $5 \mathrm{G}$, and utilising $4 \mathrm{G}$ LTE is a viable solution as a transition to $5 \mathrm{G}$ allowing the easier and potentially more affordable adoption of $5 \mathrm{G}$ when it is more mainstream.

\subsection{Fog and Edge Computing}

Edge computing is currently being implemented in many industries utilizing the industry backbone for data management known as the programmable logic controller (PLC). The main task of a PLC is to acquire data from field devices, apply logic or arithmetic functions and set output values based on these computations. This typically requires round-trip times in the range of several microseconds to milliseconds. With the use of $5 \mathrm{G}$ networks, the rate at which instructions are processed by edge networks will increase with the flow of more considerable amounts of data. Fog computing brings intelligence to the shop floor network; edge computing brings intelligence, processing power, and communication capabilities directly into devices such as smart sensors and gateway devices [2].

\subsubsection{Edge Computing}

The IIoT edge gateway, or aggregation point (AP), primarily provides connectivity to networks with a large number of sensors, controllers and actuators, all of which can use different wireless/wired technologies. It is located on the edge of the network close to the data provider and the edge of the system leading from the machine to the cloud [2,22].

In an original conceptual IoT topology, smart sensors would collect data, and send it back to the cloud for processing. One of the critical enablers for the IoT market is the proliferation of sensors and the actuator. These sensors need to be capable of many features whilst meeting the industry demands, which are mainly; the reduction the price of sensors, adding compute capability and connectivity capabilities through either their wired (RS232/422/485 etc.), wireless (Wi-Fi, SigFOX, Lora, BLE, ZigBee etc.) or radio (3G and $4 \mathrm{G} / \mathrm{LTE}$ etc.) technologies. These features will considerably increase the value of these devices, and more importantly enable real-time management of the devices especially in large sensor arrays. 
In manufacturing environments, programmable logic circuits (PLCs) already undertake the context and compute capabilities, however frequently do not have connectivity capabilities or if they do, they are proprietary in nature [23]. Conversely one of the main reasons the Internet of Things is becoming more prevalent is due to the reduced price of commodity sensors and actuators [24] adding connectivity and compute capabilities would provide pricing and implications for large sensor arrays.

Edge devices provide capabilities, correlating data from multiple input protocols and mediums, undertaking local processing, such as data compression, or decision making using artificial intelligence algorithms [25], while providing connectivity to TCP/IP via IP Networks, Wi-Fi or radio access networks (RAN) and interfacing to proprietary networking and industrial connectivity protocols. Another crucial role of edge compute devices, or gateways, is to provide continuous operation, running rulesets locally even during outages or when latency issues occur on the WAN link back to the Cloud infrastructure [26].

\subsubsection{Multi-Access Edge Computing (MEC)}

Multi-access edge computing (MEC), in the past known as mobile edge computing is a variant of edge computing that brings the capabilities of cloud computing to the edge of the network. This allows sharing of network and compute capabilities virtually anywhere, and will enable reduced deployment time and centralized management of remote compute capabilities. Whereas traditional cloud computing occurs typically on remote virtual servers that are situated far from the facility and the device, MEC allows processes to take place in aggregation points on the network, bringing decision making closer to the production environment. By opening this MEC environment up to more than just cellular users, ETSI (European Telecommunications Standards Institute) speculates that "The capabilities and characteristics offered by a MEC platform can be leveraged in a way that will allow proximity, agility and speed to be used for broader innovation that can be translated into unique value and revenue generation [27]. According to research by Tran, et al. [28,29] the use of MEC and the use of commodity server platforms to provide MEC capabilities identified five critical areas for MEC architectures to be successful; see Table 4.

Table 4. Key areas for multi-access edge computing (MEC) architectures [2].

\begin{tabular}{cl}
\hline Deployment Scenarios and Resource Management & $\begin{array}{l}\text { How to identify where the best locations to install the MEC } \\
\text { platforms physically and how to manage them remotely. }\end{array}$ \\
\hline Computational Caching and Offload & $\begin{array}{l}\text { The combination of computing and storage locally can provide } \\
\text { an increase in compute capability and speed to the user, whilst } \\
\text { reducing traffic to the cloud connections. There are challenges } \\
\text { here around deciding what to cache, as each user's computing } \\
\text { requirements may have unique data requirements. }\end{array}$ \\
\hline IoT Applications and Big Data Analytics & $\begin{array}{l}\text { MEC can help reduce and refine datasets before sending back } \\
\text { to big data platforms for analysis/deep learning. }\end{array}$ \\
\hline Security and Privacy & $\begin{array}{l}\text { Ensuring continuous connectivity for mobile users is a crucial } \\
\text { consideration for MEC, with trajectory predictions (of the user) } \\
\text { enabling the caching of data amongst MEC nodes in the } \\
\text { network. }\end{array}$ \\
\hline $\begin{array}{l}\text { As there are multiple compute points, at remote locations, the } \\
\text { attack surface is increased exponentially over a traditional } \\
\text { cloud platform. Privacy of the user's computing requirements } \\
\text { is also a key concern for MEC systems. }\end{array}$
\end{tabular}

\section{The Wireless Spectrum and 5G}

With the rapid development of wireless communication technologies, the need for bandwidth is increasing. Usage of the wireless spectrum has increased in recent years and that has resulted in the necessity to manage the spectrum to allow multiple end-user devices to communicate in a structured 
method enabling interoperability across a wide array of wireless protocols both presently available and those that are due for release in the near future [30-32].

In accordance with European law, the radio spectrum is awarded to a technology and is applied in a technology neutral manner, this means that licensees may use the radio spectrum as they see fit provided that they comply with the relevant applicable harmonized standards in the band in which they communicate. In Ireland, radio frequencies are treated as a national resource and their licensing is managed by the national communications Regulator, ComReg [33-37].

When an end user such as a company plans to deploy a wireless technology in a licensed band, the procedure for this is that the company will require a license from the government regulator to operate in the chosen spectrum, in the geographical area assigned for the deployment. It is important to note that this process only applies when operating in bands of the spectrum that are licensed. No license is required to operate in unlicensed frequency bands as long as the equipment is certified and approved for use in the jurisdiction. Examples of unlicensed bands would be the $418 \mathrm{MHz}$ and $433 \mathrm{Mhz}$ channels often used for remote controls etc. [37-39].

The development of wireless communication technology has driven the need for bandwidth, and that growing need is pushing the wireless spectrum resources to become more scarce. To address the expected growth in traffic in the next iteration of wireless networks, there is now an increasing interest in modifying the traditional allocation of the spectrum towards a more dynamic usage model, where the spectrum is treated as a shared resource which will allow for the deployment of multiple wireless networks in a heterogeneous architecture within a limited site of operation. The planned heterogeneous approach is advocating a transitional approach from the static allocation of spectrum, to a more dynamic approach, which will free up the spectrum's resources. This shared infrastructure approach could have a transformative effect on how factories deploy their wireless assets in the future. Spectrum sharing in future wireless networks will likely occur in both licensed bands (e.g., the 2.3-2.4 GHz band in Europe and the 3.55-3.65 GHz band in the USA) and unlicensed bands [40-43]. A summary of the main wireless protocols and their attributes is in Table 5.

Table 5. Comparison of wireless protocols.

\begin{tabular}{cccc}
\hline Protocol & Frequency & Wavelength & Range \\
\hline Wi-Fi (2.4G) & $2401 \mathrm{MHz}-2483 \mathrm{MHz}$ & $12 \mathrm{~cm}$ & $150 \mathrm{~m}$ \\
\hline Wi-Fi (5G) & $5150 \mathrm{MHz}-5875 \mathrm{MHz}$ & $5 \mathrm{~cm}$ & $120 \mathrm{~m}$ \\
\hline Bluetooth & $2400 \mathrm{MHz}-2485 \mathrm{MHz}$ & $12 \mathrm{~cm}$ & $10-100 \mathrm{~m}$ \\
\hline LoRA & $868 \mathrm{MHz}$ & $35 \mathrm{~cm}$ & $10 \mathrm{~km}+$ \\
\hline $3 G$ & $900 / 2100 \mathrm{MHz}$ & $33.3 / 14 \mathrm{~cm}$ & $100 \mathrm{~m}$ to $>5 \mathrm{~km}$ \\
\hline $4 \mathrm{G}$ (LTE) & $800 / 1800 \mathrm{MHz}$ & $37.5 / 17 \mathrm{~cm}$ & $100 \mathrm{~m}$ to $>5 \mathrm{~km}$ \\
\hline $5 G$ & $700 \mathrm{MHz} / 3.6 \mathrm{GHz} / 26$ & $43 \mathrm{~cm} / 8 \mathrm{~cm} / 1 \mathrm{~mm}$ & $50 \mathrm{~m}$ to $>5 \mathrm{~km}$ \\
\hline
\end{tabular}

Communications regulators globally are managing the allocation of spectrum for $5 \mathrm{G}$ usage; typically, the spectrum is being auctioned to telcos who are then offering a range of solutions to industry. In Europe, three major frequency bands have been made available for $5 \mathrm{G}$ use. These three bands are $700 \mathrm{MHz}, 3.6 \mathrm{GHz}$ and $26 \mathrm{GHz}$ as shown in Table 5 .

When considering the heterogeneous approach to wireless networks it is important to understand that interaction of the wireless spectrum with cellular technologies will be a dominant driver for many sites. It should be noted that spectrum regulators in some countries have started the process of opening up shared spectrum for local use in specific bands, which will enable the deployment of private 5G networks and enable easier research and development deployments. For example, the regulator in the USA opened up the $3.5 \mathrm{GHz}$ band as of January 2020 for commercial use known as the CBRS (Citizens Broadband Radio Service) band. 


\section{Private 5G Networks}

For many of the world's businesses, private $5 \mathrm{G}$ will likely become the preferred choice, especially for industrial environments such as, manufacturing plants, logistics centres, and ports. A mobile private 5G network, as the name suggests, is a local area network that utilises $5 \mathrm{G}$ technology as its communication medium to build and create a 'private' network.

Private 5G networks are in some circumstances referred to as local 5G networks or mobile private networks (MPN), and offer unified connectivity with various advantages and optimized services. A private network that is created for an organisation is expected to carry all the features of $5 \mathrm{G}$ public networks, including the reduced latency and higher speeds. Deploying a private network delivers several advantages in terms of efficiency and security this is why companies, regulators, organizations and other potential users are currently looking ahead to the arrival of private $5 \mathrm{G}$ networks to get the most out of this technology. Private 5G networks have the potential to become the future communication platform of the factory as they will be able to provide for the increase in bandwidth requirements coming from more connected equipment and the corresponding data being transmitted at the factory floor, as well as the millisecond latency required for real-time remote control of robotics.

Since private 4G LTE networks are already commercial and in extensive use, deploying private $5 \mathrm{G}$ networks is obviously an expectation that will allow manufacturers to eliminate the tethered limitations of ethernet cables that litter their factory floors and connect the machines wirelessly to the cloud. The capacity of a network due to the number of IoT and wireless devices to be connected on a network will be considerably higher once a 5G network is deployed due to the increased bandwidth of 5G [44]. Due to the wide variations of IoT requirements, several overlapping topologies exist, from the sensor or actuator through local environments, to decentralized or core cloud capabilities, and reaching out to public, private or hybrid clouds [45]. A significant challenge for $5 \mathrm{G}$ in business terms is if the plant owners and operators are willing to pay for a licensed spectrum instead of using available unlicensed spectrum technology, which is effectively a potential cost-free improvement. To enable enterprise connectivity with ultra-reliable, high-speed, low-latency, power-efficient, high-density wireless connectivity a company essentially has two options:

- Connect to a public 5G network.

- $\quad$ Build and use a private 5G network.

Deployments in industry have been mainly in the two formats mentioned above. These involve the deployment of a physically-isolated private 5G network (a 5G island) that is independent of the mobile operator's public $5 \mathrm{G}$ network. Audi, for example, has already started testing $5 \mathrm{G}$ for robotic motion control and Nokia is using $5 \mathrm{G}$ for in-factory private $5 \mathrm{G}$ connectivity. Another example of the private network deployment model is the e.GO car company in Aachen, Germany. In e.GO's factory, where the e.GO Life model is manufactured, an Ericsson private network with a 5G core and $5 \mathrm{G}$ new radio solution has been deployed to deliver secure and near real-time data networking across the production chain, from digital material management to autonomous vehicle control. The on-site private 5G network has helped establish an automated factory realizing and delivering connectivity for an array of connected devices such as machines, sensors, materials and robots can be managed through one standardized network, with the correct latency and bandwidth allocated as necessary.

As 5G has developed it has been adopted by companies eager to test and potentially take first mover advantage. The second deployment scenario being adopted by companies is a more basic approach, to connect to a public 5G network, sharing the mobile operator's public 5G network with other users. An example of this scenario is when companies are connecting to the $5 \mathrm{G}$ network due to inadequate Wi-Fi connectivity options. In both deployment models; for 5G to be successful in a manufacturing environment there must be a close collaboration between all systems, spanning across the corporate IT and the manufacturing OT to maximize the capabilities inherent in 5G [46-49].

For deployment of a private $5 \mathrm{G}$ network it would involve a company purchasing an infrastructure while contracting for operational support usually from a mobile operator. Or the company can build 
and maintain their own 5G network using their own specific spectrum, obtained from the spectrum regulator in the country where the $5 \mathrm{G}$ network will operate. An example of a proposed layout for $4 \mathrm{G} / 5 \mathrm{G}$ deployment in a corporate network is shown in Figure 5.

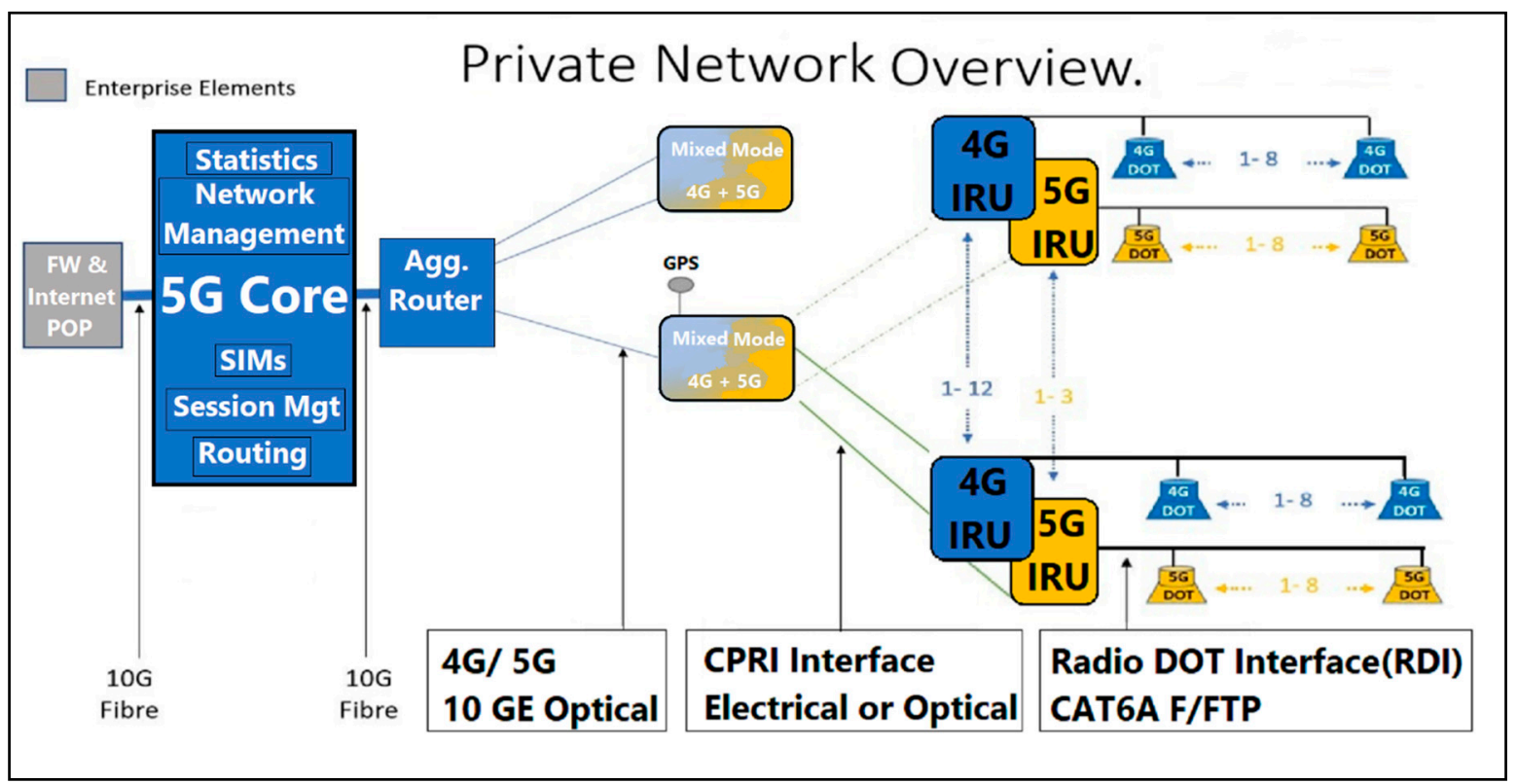

Figure 5. Private 5G infrastructure supporting Industry 4.0.

\section{Benefits of Private 5 G Networks}

Non-public 5G networks turn businesses into mobile operators in limited geographic areas. A company that operates a mobile private $5 \mathrm{G}$ network (MPN) will become a $5 \mathrm{G}$ network infrastructure operator; this will probably be the case if the organisation wants to have complete control over its operating processes. As a result, parameters such as the bandwidth or real-time properties can be adapted, which is not possible when using the public network of a mobile radio provider.

An organisation that runs a private $5 \mathrm{G}$ network will be able to add layered security at every level of the manufacturing process. Since the security can be higher, it will afford the owner of the network a level of control that would not be possible on a public network. These networks also offer protection against industrial espionage. Data in non-public 5G networks can be segregated and processed separately from public $5 \mathrm{G}$ networks which will ensure complete privacy protection of process and production-related data because the communication takes place within a separate network, enabling the data to be far better protected against unauthorised access [50,51].

Unlike a public network, a private 5G network can be configured to the specific needs of a location, and configurations can vary by site, depending on the type of work undertaken in each venue. A private network allows companies to determine the network's deployment timetable and coverage quality. Another point of note is that the network may be installed and maintained by onsite personnel, enabling faster responses to issues, thereby reducing downtime and maintaining a level of uptime to meet the organisation's needs.

\section{Security in the Wireless Factory of the Future}

One of the initial concerns that companies have about deploying a 5G network to a site that will have connectivity to thousands of devices is security, because 5G connected devices will dramatically expand the potential network-attack surface, Figure 6. A smart factory's system will include countless pieces of equipment and devices that are connected to a single organisational network. Vulnerabilities in any one of those devices can create a gap in the network that could open the system up to security threats. From a factory viewpoint, OT systems have predominantly been isolated from external networks 
for many decades. However, due to the introduction of TCP/IP and other network communication standards within OT environments, the OT elements have become susceptible to cyber-attacks in recent years [52,53].

As factories of the future transition increasingly towards wireless infrastructures, both currently available and due for release, they will have to build a comprehensive cybersecurity plan that addresses the threats resulting from IT/OT convergence, such as:

- Denial-of-service (DoS) and distributed denial-of-service (DDoS) attacks on production systems.

- Man-in-the-middle attack for production data theft due to gaps in the IT structure of the newly adapted manufacturing processes.

- Anomalies based on human behaviour and errors.

- Critical system access to third-party contractors.

- Legacy devices with limited or no security services such as programmable logic controllers (PLCs), remote terminal units (RTUs), supervisory control and data acquisition (SCADA) servers, integrated with fully accessible and network-enabled IoT devices.

- $\quad$ Lack of adequate visibility and cyber-resilient systems. Traditionally OT systems have achieved security by obscurity and this is no longer a guarantee of protection with the advent of wireless connectivity and massive machine-type communication (mMTC).

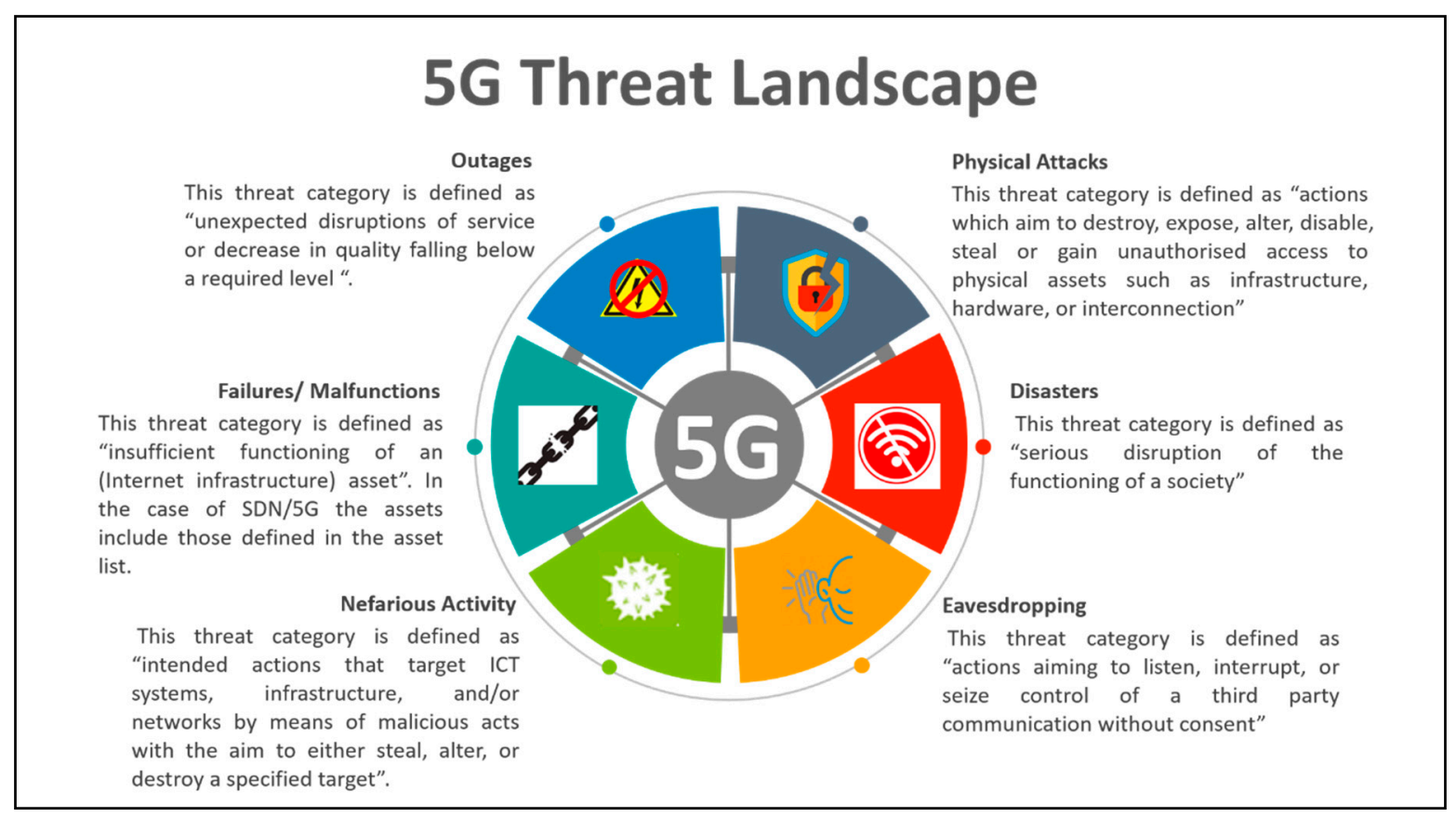

Figure 6. The $5 \mathrm{G}$ threat landscape.

Historically, successful transitions into new technologies have always been focused on end goal results with security rarely at the forefront. While $5 \mathrm{G}$ has incorporated security into its design a logical approach for smart manufacturing should be to develop a more robust suite of security protocols at the outset [54]. This will allow for an easier adoption by manufacturers and for a more robust end user system that would allow for multiple wireless technologies operating in parallel across a production floor. As giving a level of trustworthiness in the system to meet regulatory requirements will be an issue when systems are deployed in regulated manufacturing environments, a model that demonstrates trustworthiness and reliability will need to be honed for the manufacturing sector. 


\section{Standards and Industry Associations}

The 5G wireless standard aims to be global which is difficult due to different implementation approaches in multiple countries, e.g., Russia, China and South Korea or an amalgamated body of countries (e.g., the European Union (EU)) outlining their own definition of 5G networks, speeds and regulations outlining where $5 \mathrm{G}$ transmissions may occur. In broad terms, standards benefit innovation, the environment, safety and the economy. Many standards bodies are contributing to the development of 5G for manufacturing, e.g., The 5G Alliance for Connected Industries and Automation (5G-ACIA) is a global forum for collaboration. A summary list is given below.

- Industrial Internet Consortium (IIC), https://www.iiconsortium.org/index.htm.

- The 3rd Generation Partnership Project (3GPP), https://www.3gpp.org/.

- The 5G Alliance for Connected Industries and Automation (5G-ACIA), https://www.5gacia.org/ about-5g-acia/.

- $\quad$ EU 5GPPP, https://5g-ppp.eu/.

Industry, whilst working in conjunction with standards, has many open questions still to investigate, such as:

- Co-existence of different wireless protocols and systems;

- Co-existence of different wired protocols;

- Interoperability between wired and wireless communication systems;

- Seamless engineering taking into account collected real-life data;

- Missing capabilities for plug-and-produce integration of sensors, machinery and people;

- Missing capabilities for cost-effective network and service management by factory operator;

- Managing workflows and data interaction patterns between an increasing number of sensors, machines, robots, wearables, etc.;

- Allocating the proper computing resources in the cloud, on the edge or at sensor levels to ensure compliance with application-level service agreements;

- Leveraging machine-learning and data analytics capabilities distributed in the network, to a multitude of vendor-specific platforms, contributing to a unified, intelligent data intelligence platform;

- Managing and optimizing the wireless network topology and performance, according to real-time changing networking conditions.

Future Improvements of $5 G$ towards $6 G$

While 5G will be improved upon, 6G is expected to be launched commercially in 2030. It is a technology that is being developed in response to the increasing availability of distributed radio access network (RAN) and the desire to take advantage of the terahertz spectrum (THz) to further increase capacity and reduce latency. It is expected that many of the perceived problems associated with deploying millimeter-wave radio for $5 \mathrm{G}$ radio will be resolved in time for network designers to address the challenge of implementing a 6G offering. $6 \mathrm{G}$ connectivity is expected to support speeds of 1 terabyte per second (Tbps). This level of capacity and latency will be unprecedented and will extend the performance of $5 \mathrm{G}$ applications along with expanding the scope of capabilities in support of increasingly new and innovative tools in the areas of wireless cognition, detection and imaging. In addition, 6G wireless sensing solutions are expected to selectively use different frequencies to measure absorption and adjust frequencies accordingly in order to maximize signal penetration and transmission distances.

\section{Conclusions}

All of the technological and business monitors are indicating that $5 \mathrm{G}$ has the potential to revolutionize manufacturing and, over time, will improve productivity. While 5G is capable of bringing 
many solutions, there is also a myriad of engineering matters that need to be considered before 5G can be the all-conquering panacea for manufacturing. While there are many barriers to entry for industry adopting the suite of technologies incorporated in 5G, ranging from, spectrum allocation, scalability issues, edge analytics and security concerns which have been detailed in this paper, further investigation is required into the adoption of an unbounded wireless transmission medium in a society that is increasingly conscious of data privacy and that will be a challenge not just for engineers but also standards bodies and lawmakers.

When considering a 5G deployment, industry needs to assess the specific requirements for each IoT use case. Industry needs to get a clearer understanding of the data types being transmitted, their sensitivity to latency, and whether the data needs to be processed by itself, or against historical information. The answers to these requirements and other factors that will dictate whether or not a company needs to implement a smart manufacturing solution using $5 \mathrm{G}$ topologies or whether there are other protocols that may be more suited to the individual solution.

$5 \mathrm{G}$ will not only advance manufacturing operations by enabling businesses to be faster but will also make them more responsive to customer needs. Manufacturers will benefit from the deployment of 5G since warehouses, factories and other facilities will increasingly have been enabled with smart technologies that will benefit from higher speed networks. 5G's unprecedented speed and coverage will bring the world closer than ever, as well as providing an avenue for never before imagined capabilities across the manufacturing space. It is an exciting time for manufacturing and for any company that can leverage the improvements that $5 \mathrm{G}$ and beyond offers.

Finally, it is clear that further research should be undertaken into the interoperability of wireless technologies in factory environments to reveal the reliability, latency, and minimum range of wireless technologies in a factory environment when deployed in conjunction with 5G, evaluating the heterogeneous interoperability of $5 \mathrm{G}$ and existing communication protocols currently deployed in manufacturing environments.

Funding: The authors would also like to acknowledge resources made available through the Science Foundation Ireland through the grant award (16/RC/3918) to the Confirm Centre for Smart Manufacturing.

Acknowledgments: The authors would like to thank the staff of the Electronic and Computer Engineering Department at the University of Limerick for their assistance in this work.

Conflicts of Interest: The authors declare no conflict of interest.

\section{References}

1. Mao, Y.; You, C.; Zhang, J.; Huang, K.; Letaief, K.B. A Survey on Mobile Edge Computing: The Communication Perspective. IEEE Commun. Surv. Tutor. 2017, 19, 2322-2358. [CrossRef]

2. McCann, J.; Quinn, L.; McGrath, S.; O'Connell, E. Towards the Distributed Edge-An IoT Review. In Proceedings of the 2018 12th International Conference on Sensing Technology (ICST), Limerick, Ireland, 3-6 December 2018; pp. 263-268.

3. Bockelmann, C.; Pratas, N.K.; Wunder, G.; Saur, S.; Navarro, M.; Gregoratti, D.; Vivier, G.; De Carvalho, E.; Ji, Y.; Stefanovic, C.; et al. Towards Massive Connectivity Support for Scalable mMTC Communications in 5G Networks. IEEE Access 2018, 6, 28969-28992. [CrossRef]

4. Popovski, P.; Trillingsgaard, K.F.; Simeone, O.; Durisi, G. 5G Wireless Network Slicing for eMBB, URLLC, and mMTC: A Communication-Theoretic View. IEEE Access 2018, 6, 55765-55779. [CrossRef]

5. Siddiqi, M.; Yu, H.; Joung, J. 5G Ultra-Reliable Low-Latency Communication Implementation Challenges and Operational Issues with IoT Devices. Electronics 2019, 8, 981. [CrossRef]

6. Gidlund, M.; Lennvall, T.; Akerberg, J. Will 5G become yet another wireless technology for industrial automation? In Proceedings of the 2017 IEEE International Conference on Industrial Technology (ICIT), Toronto, ON, Canada, 22-25 March 2017; pp. 1319-1324.

7. Orlosky, J.; Kiyokawa, K.; Takemura, H. Virtual and Augmented Reality on the 5G Highway. J. Inf. Process. 2017, 25, 133-141. [CrossRef] 
8. GSMA Intelligence. Understanding 5G: Perspectives on Future Technological Advancements in Mobile. White Paper. 2014, pp. 1-26. Available online: https://www.gsma.com/futurenetworks/wp-content/uploads/ 2015/01/2014-12-08-c88a32b3c59a11944a9c4e544fee7770.pdf (accessed on 10 June 2020).

9. Cheng, J.; Chen, W.; Tao, F.; Lin, C.-L.; Da Xu, L. Industrial IoT in 5G environment towards smart manufacturing. J. Ind. Inf. Integr. 2018, 10, 10-19. [CrossRef]

10. Mudrakola, S. Private 5G Networks. 2019. Available online: http://techgenix.com/private-5g-networks/ (accessed on 10 June 2020).

11. Onoe, S. Plenary speaker 1. In Proceedings of the 2017 IEEE Radio Frequency Integrated Circuits Symposium (RFIC), Honolulu, HI, USA, 4-6 June 2017; p. 1. [CrossRef]

12. Atanasovski, V.; Leon-Garcia, A. Future Access Enablers for Ubiquitous and Intelligent Infrastructures; Springer: Berlin, Germany, 2015.

13. Baharom, B.; Ali, M.T. Multiple-element PIFA MIMO antenna system design for future 5G wireless communication applications. In Proceedings of the 2017 IEEE Asia Pacific Microwave Conference (APMC), Kuala Lumpur, Malaysia, 13-16 November 2017; pp. 743-746.

14. Arthur D. Little for Vodafone Group Plc. "Creating a Gigabit Society". Available online: https:/www.vodafone.com/content/dam/vodcom/files/public-policy/Vodafone_Group_Call_for_ the_Gigabit_SocietyFV.pdf (accessed on 10 June 2020).

15. Rampa, V.; Savazzi, S.; Malandrino, F. Opportunistic sensing in beyond-5G networks: The opportunities of transformative computing. In 5G Italy Book 2019: A Multiperspective View of 5G; CNIT: Parma, Italy, 2019; pp. 461-476. ISBN 9788832170030.

16. Wdowik, R.; Ratnayake, R.C. Collaborative Technological Process Planning with 5G Mobile Networks and Digital Tools: Manufacturing Environments' Perspective. In Proceedings of the 2019 IEEE International Conference on Industrial Engineering and Engineering Management (IEEM), Macau, China, 15-18 December 2019; pp. 349-353.

17. Plant Automation Technology Impact of 5G in Manufacturing Industry. Available online: https: //www.plantautomation-technology.com/articles/impact-of-5g-in-manufacturing-industry (accessed on 10 June 2020).

18. Elayoubi, S.E.; Fallgren, M.; Spapis, P.; Zimmermann, G.; Martín-Sacristán, D.; Yang, C.; Jeux, S.; Agyapong, P.; Campoy, L.; Qi, Y.; et al. 5G service requirements and operational use cases: Analysis and METIS II vision. In Proceedings of the 2016 European Conference on Networks and Communications (EuCNC), Athens, Greece, 27-30 June 2016; pp. 158-162.

19. Singh, S.; Singh, P. Key concepts and network architecture for $5 \mathrm{G}$ mobile technology. Int. J. Sci. Res. Eng. Technol. (IJSRET) 2012, 1, 165-170.

20. Barakabitze, A.A.; Ahmad, A.; Mijumbi, R.; Hines, A. 5G network slicing using SDN and NFV: A survey of taxonomy, architectures and future challenges. Comput. Netw. 2020, 167, 106984. [CrossRef]

21. Chen, B.; Wan, J.; Shu, L.; Li, P.; Mukherjee, M.; Yin, B. Smart Factory of Industry 4.0: Key Technologies, Application Case, and Challenges. IEEE Access 2018, 6, 6505-6519. [CrossRef]

22. Ludwig, S.; Karrenbauer, M.; Fellan, A.; Schotten, H.; Buhr, H.; Seetaraman, S.; Niebert, N.; Bernardy, A.; Seelmann, V.; Stich, V.; et al. A5G architecture for the factory of the future. In Proceedings of the 2018 IEEE 23rd international conference on emerging technologies and factory automation (ETFA), Torino, Italy, 4-7 September 2018; Volume 1, pp. 1409-1416.

23. Shi, W.; Cao, J.; Zhang, Q.; Li, Y.; Xu, L. Edge Computing: Vision and Challenges. IEEE Internet Things J. 2016, 3, 637-646. [CrossRef]

24. Gubbi, J.; Buyya, R.; Marusic, S.; Palaniswami, M. Internet of Things (IoT): A vision, architectural elements, and future directions. Futur. Gener. Comput. Syst. 2013, 29, 1645-1660. [CrossRef]

25. Vermesan, O.; Bröring, A.; Tragos, E.; Serrano, M.; Bacciu, D.; Chessa, S.; Gallicchio, C.; Micheli, A.; Dragone, M.; Saffiotti, A.; et al. Internet of robotic things: Converging sensing/actuating, hypoconnectivity, artificial intelligence and IoT Platforms. In Cognitive Hyperconnected Digital Transformation: Internet of Things Intelligence Evolution; River Publishers: Gistrup, Denmark, 2017; pp. 97-155.

26. Gremban, K. Editorial and Introduction to the Issue: Risk and Rewards of the Internet of Things. IEEE Internet Things Mag. 2018, 1, 2-3. [CrossRef]

27. Hu, Y.C.; Patel, M.; Sabella, D.; Sprecher, N.; Young, V. Mobile edge computing-A key technology towards 5G. ETSI White Paper 2015, 11, 1-16. 
28. Tran, T.X.; Hajisami, A.; Pandey, P.; Pompili, D. Collaborative Mobile Edge Computing in 5G Networks: New Paradigms, Scenarios, and Challenges. IEEE Commun. Mag. 2017, 55, 54-61. [CrossRef]

29. Tran, T.X.; Hosseini, M.-P.; Pompili, D. Mobile edge computing: Recent efforts and five key research directions. IEEE COMSOC MMTC Commun-Frontiers 2017, 12, 29-34.

30. Wu, Q.; Ding, G.; Du, Z.; Sun, Y.; Jo, M.; Vasilakos, A.V. A Cloud-Based Architecture for the Internet of Spectrum Devices Over Future Wireless Networks. IEEE Access 2016, 4, 2854-2862. [CrossRef]

31. Wu, C.; Wang, Y.; Yin, Z. Energy-efficiency opportunistic spectrum allocation in cognitive wireless sensor network. EURASIP J. Wirel. Commun. Netw. 2018, 2018, 1-14. [CrossRef]

32. Wieruch, D.; Holfeld, B.; Wirth, T. Wireless Factory Automation: Radio Channel Evolution in Repeated Manufacturing Processes. In Proceedings of the WSA 2016, 20th International ITG Workshop on Smart Antennas, VDE, Munich, Germany, 9-11 March 2016; pp. 1-4.

33. Montgomery, K.; Candell, R.; Liu, Y.; Hany, M. Wireless User Requirements for the Factory Workcell. Available online: https://tsapps.nist.gov/publication/get_pdf.cfm?pub_id=928517 (accessed on 10 June 2020).

34. Mochón, M.A.; Saez, Y. A review of radio spectrum combinatorial clock auctions. Telecommun. Policy 2017, 41, 303-324. [CrossRef]

35. Kalliovaara, J.; Ekman, R.; Paavola, J.; Jokela, T.; Hallio, J.; Auranen, J.; Talmola, P.; Kokkinen, H. Designing a testbed infrastructure for experimental validation and trialing of $5 \mathrm{G}$ vertical applications. In Proceedings of the International Conference on Cognitive Radio Oriented Wireless Networks, Lisbon, Portugal, 20-21 September 2017; pp. 247-263. [CrossRef]

36. Hu, F.; Chen, B.; Zhu, K. Full Spectrum Sharing in Cognitive Radio Networks Toward 5G: A Survey. IEEE Access 2018, 6, 15754-15776. [CrossRef]

37. Ejaz, W.; Ibnkahla, M. Multiband Spectrum Sensing and Resource Allocation for IoT in Cognitive 5G Networks. IEEE Internet Things J. 2017, 5, 150-163. [CrossRef]

38. Doyle, L.; Kibilda, J.; Forde, T.K.; DaSilva, L. Spectrum Without Bounds, Networks Without Borders. Proc. IEEE 2014, 102, 351-365. [CrossRef]

39. Dong, X.; Cheng, L.; Zheng, G.; Wang, T. Network access and spectrum allocation in next-generation multi-heterogeneous networks. Int. J. Distrib. Sens. Netw. 2019, 15, 1550147719866140. [CrossRef]

40. IEEE. 802.11-2016-IEEE Standard for Information Technology-Telecommunications and Information Exchange between Systems Local and Metropolitan Area Networks-Specific Requirements-Part 11: Wireless LAN Medium Access Control (MAC) and Physical Layer (PHY) Specifications; IEEE: Piscataway, NJ, USA, 2016.

41. Bhattarai, S.; Park, J.-M.; Gao, B.; Bian, K.; Lehr, W. An Overview of Dynamic Spectrum Sharing: Ongoing Initiatives, Challenges, and a Roadmap for Future Research. IEEE Trans. Cogn. Commun. Netw. 2016, 2, 110-128. [CrossRef]

42. Ashraf, S.A.; Aktas, I.; Eriksson, E.; Helmersson, K.W.; Ansari, J. Ultra-reliable and low-latency communication for wireless factory automation: From LTE to 5G. In Proceedings of the 2016 IEEE 21st International Conference on Emerging Technologies and Factory Automation (ETFA), Berlin, Germany, 6-9 September 2016; pp. 1-8. [CrossRef]

43. Akyildiz, I.F.; Lee, W.-Y.; Vuran, M.C.; Mohanty, S. NeXt generation/dynamic spectrum access/cognitive radio wireless networks: A survey. Comput. Netw. 2006, 50, 2127-2159. [CrossRef]

44. Mahmud, R.; Kotagiri, R.; Buyya, R. Fog Computing: A Taxonomy, Survey and Future Directions. In Internet of Everything; Springer: Berlin, Germany, 2018; pp. 103-130.

45. Li, J.; Li, Y.K.; Chen, X.; Lee, P.P.; Lou, W. A Hybrid Cloud Approach for Secure Authorized Deduplication. IEEE Trans. Parallel Distrib. Syst. 2014, 26, 1206-1216. [CrossRef]

46. Chien, H.-T.; Lin, Y.-D.; Lai, C.-L.; Wang, C.-T. End-to-End Slicing as a Service with Computing and Communication Resource Allocation for Multi-Tenant 5G Systems. IEEE Wirel. Commun. 2019, 26, 104-112. [CrossRef]

47. Coll-Perales, B.; Gozalvez, J.; Maestre, J.L. 5G and Beyond: Smart Devices as Part of the Network Fabric. IEEE Netw. 2019, 33, 170-177. [CrossRef]

48. Gringoli, F.; Patras, P.; Donato, C.; Serrano, P.; Grunenberger, Y. Performance Assessment of Open Software Platforms for 5G Prototyping. IEEE Wirel. Commun. 2018, 25, 10-15. [CrossRef]

49. Di Mauro, M.; Liotta, A. An experimental evaluation and characterization of VoIP over an LTE-A network. IEEE Trans. Netw. Serv. Manag. 2020. [CrossRef] 
50. O'Connell, E.; Healy, M.; O'Keeffe, S.; Newe, T.; Lewis, E. Development of a discriminating fibre optic sensing array for wireless real time analysis of the maritime environment. In Proceedings of the OFS2012 22nd International Conference on Optical Fiber Sensors, Beijing, China, 14-19 October 2012. [CrossRef]

51. Ruili, J.; Haocong, W.; Han, W.; O'Connell, E.; McGrath, S. Smart Parking System Using Image Processing and Artificial Intelligence. In Proceedings of the 2018 12th International Conference on Sensing Technology (ICST), Limerick, Ireland, 3-6 December 2018; pp. 232-235.

52. Haerick, W.; Gupta, M. 5G and the Factories of the Future. 5G-PPP White Paper. 2015. Available online: https://5g-ppp.eu/wp-content/uploads/2014/02/5G-PPP-White-Paper-on-Factories-of-the-FutureVertical-Sector.pdf (accessed on 10 June 2020).

53. ENISA draws Threat Landscape of 5G Networks. Available online: https://www.enisa.europa.eu/news/enisanews/enisa-draws-threat-landscape-of-5g-networks. (accessed on 10 June 2020).

54. Vidal, J.M.; Monge, M.A.S. Framework for Anticipatory Self-Protective 5G Environments. In Proceedings of the 14th International Conference on Availability, Reliability and Security, Canterbury, UK, 26-29 August 2019; pp. 1-9.

C 2020 by the authors. Licensee MDPI, Basel, Switzerland. This article is an open access article distributed under the terms and conditions of the Creative Commons Attribution (CC BY) license (http://creativecommons.org/licenses/by/4.0/). 